\title{
Preclinical and preliminary clinical reports for successful treatment of ovarian cancer with standard treatments in combination with epigenetic modifiers in a complimentary and integrative approach
}

\author{
Mohammad Nezami ${ }^{1 *}$ and Jessica Garner ${ }^{2}$ \\ ${ }^{1} \mathrm{MD}$, Pacific Medical Center of Hope, Fresno, CA, USA \\ ${ }^{2}$ Department of Integrative Oncology, Orange Coast Medical Center of Hope Inc., USA
}

\begin{abstract}
Ovarian cancer is relatively uncommon, but it is extremely deadly. There are about 22,000 new cases of ovarian cancer each year, and nearly 14,000 die. Ovarian cancer possible remission happens in less than 5 percent at stage four. Less than 15 percent have a survival beyond 5 years, despite best standard treatments currently available. Here we present both invitro and invivo data to support application of a novel combinational treatment for this deadly disease. Our data (obtained in collaboration with M D Anderson) in 3-dimensional cell culture models showed inhibition of ovarian cancer cell migration and invasion. Same treatment through compassionate use in 7 cases of stage four ovarian cancer showed a positive response to a combinational therapy consisting chemotherapy, PARP inhibitors and epigenetic modifier quercetin along with other natural compounds. All seven patients had improved quality of life measured by ECOG performance scale/scores and enhanced response to conventional therapies. Although some cases did not follow through the recommended program, to accurately measure their survival, the surrogate markers for their survival improved with such therapy. We propose that such case study could generate hypothesis in further responsible use of safe and effective natural therapies outlined here.
\end{abstract}

\section{Background}

\section{Silent and deadly}

Current standard of care when used as first line treatment has shown only marginal benefit for ovarian cancer, until the disease recurs. Ovarian cancer has a recurrence of 95 percent in advanced stages. Patients who can tolerate the toxicity of the therapy initially, mainly either would become unable to tolerate the toxicity or the tumor simply becomes resistant. This has classified recurrence less than 6 months as platinum/treatment resistant. Chemo intolerance (mainly secondary to cis platinum toxicity) is commonly experienced as neuropathy in hands and feet. Considering these limitations in standard of care, there is significant research interest to identify ideal chemosensitizers to improve the response and reduce the chances of resistance and recurrence, at the same time improve patients' treatment tolerance. That said, the translational research has only provided minimal impact in clinical standards of care in stage four disease. For example, the advantages of angiogenic blockers thought initially to reduce the recurrence has been questioned as their effect is not sustained and progression free survivals seen uncommonly translate to a significantly better overall survival. One main reason is the activation of feedback loops between the VEGF and HIF-1. Also therapies that target HIF-1 are not commercially available or they are too toxic. Here we decided to collaboratively work with M D Anderson cancer center and design a treatment approach that could aim to resolve the current limitations by application of natural compounds to the ovarian cancer cell lines in specific human predictable model, assess their ability to inhibit these cells growth effectively, and further prove the concept in human by offering it, in conjunction with available standard of care, to otherwise terminal patients consensually and compassionately. These models are verified in the literature to correlate with best predictable outcome $[1,2]$. The rationale of selecting these therapies were based on current understanding of the role of histone acetylation on ovarian cancer progression and chemoresistance and their epigenetic (histone deacetylase inhibition) nature of such therapies [3-7]. Such therapies have been safe in human and several studies published on their safety, both orally and intravenously. Further response to therapy was assessed by measuring circulating tumor cells (CTC), tumor markers and imaging. Circulating tumor cell assays have shown to be predictive of overall survival and specifically the mRNA expression of epithelial markers (EpCAM) have been linked with outcome [4-9].

\section{Methods and materials}

\section{Invitro data}

Inhibition of ovarian cancer cell lines viability, both at Hey8 and OVCAR8 (with most aggressive features, and chemoresistance) were verified by epigenetic treatment at experimental therapeutics laboratory at MD Anderson (Figures 1 and 2).

${ }^{\star}$ Correspondence to: Mohammad Nezami, MD, Pacific Medical Center of Hope, Fresno, CA, USA. E-mail: amnezami@yahoo.com

Received: February 15, 2019; Accepted: March 01, 2019; Published: March 05, 2019 
Nezami M (2019) Preclinical and preliminary clinical reports for successful treatment of ovarian cancer with standard treatments in combination with epigenetic modifiers in a complimentary and integrative approach

\section{Inhibition of Migration HeyA8}
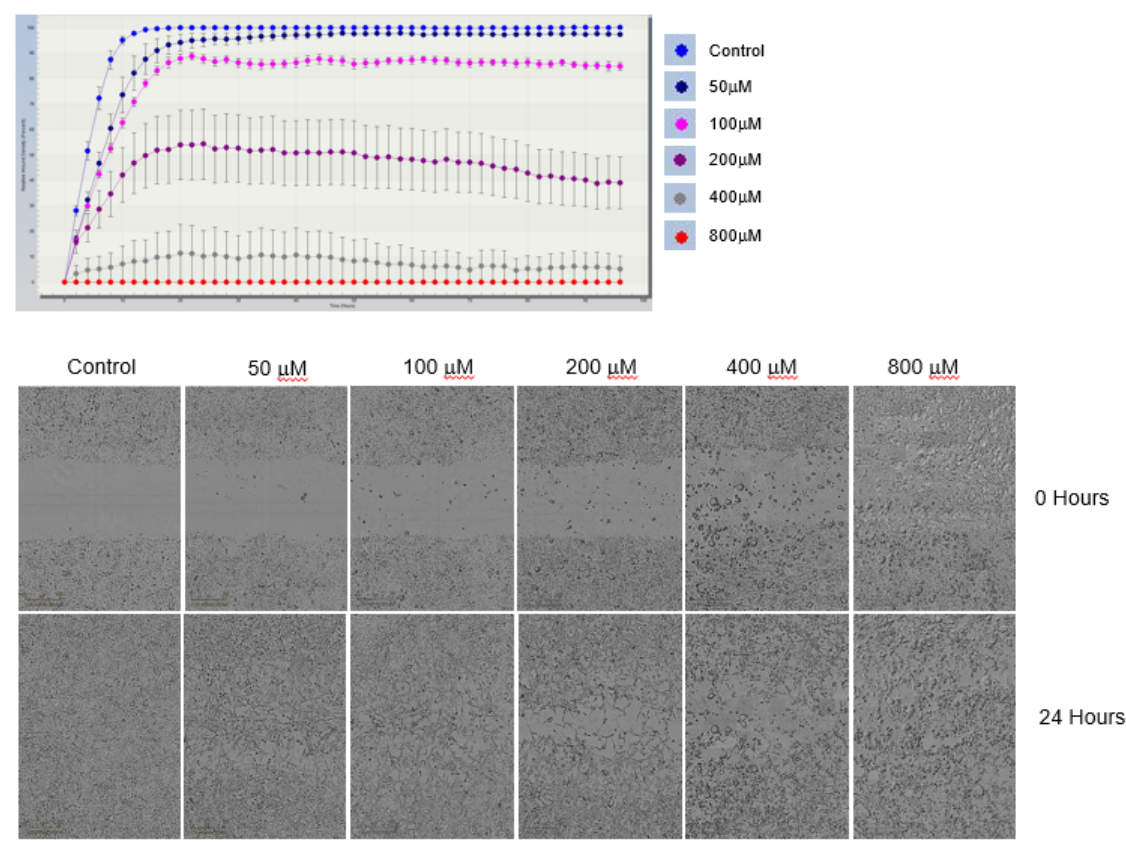

Figure 1. Live cell migration was monitored by the Incucyte Zoom over a period of 48 hours with images taken every 2 hours. We see this biological effect. Data clearly shows and inhibition of migration of the ovarian cancer cells HeyA8 by compound-Quercetin

\section{A-Viability of 3D spheroids}

OVCAR8

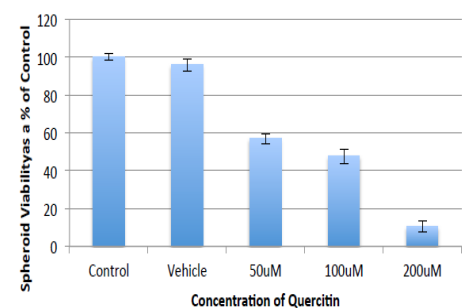

Control Venide $\quad$ SolinQ

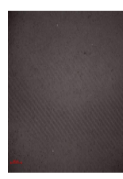

$100 \mathrm{me}$

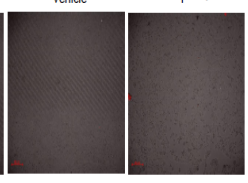

200410

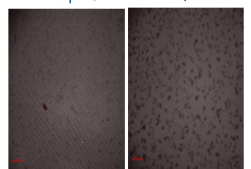

Ovary

\section{A - Viability of 3D spheroids}

HeyA8

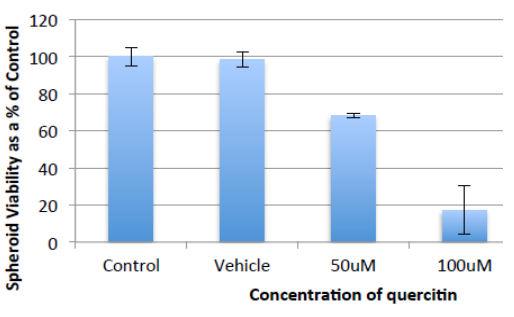

Contro

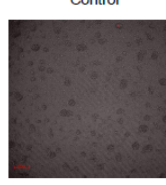

$100 \mathrm{Mm} \mathrm{O}$

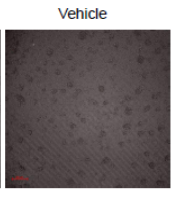

$200 \mu M$
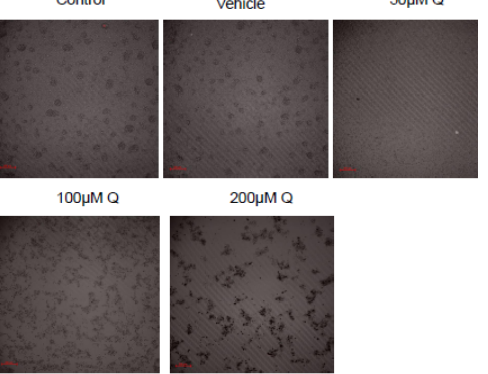

Figure 2. Destruction of two different ovarian cancer cell lines by the therapy ( 3 dimensional cell culture models show reduced viability of both Hey8 and OVCAR8 cell lines, at different concentrations of therapy

\section{Assays}

Circulating Tumor Cell (CTC) analysis was performed via Biofocus (www.biofocus.de). Heparinized blood samples of patients were processed. In brief, CTCs from $30 \mathrm{ml}$ blood were enriched by filtration cytometry using $20 \mu \mathrm{m}$ polyester filter meshes (Reichelt Chemietechnik, Heidelberg, Germany). RNA was extracted from cells retained on filter meshes with Trizol reagent. For proof of CTCs in these cell preparations, qRT-PCR for relative mRNA expression of a set of four genes (telomerase, ERBB2, c-KIT, EGFR) was performed. Assays were purchased (telomerase: Hs00972649, Applied Biosystems) or designed in-house taking care that fluorescence probes are spanning exon-boundaries. Expression values in the enriched CTC preparation 
Nezami M (2019) Preclinical and preliminary clinical reports for successful treatment of ovarian cancer with standard treatments in combination with epigenetic modifiers in a complimentary and integrative approach

was normalized to the house-keeping gene GAPDH and compared to GAPDH-normalized expression values in mononuclear cells of the patients. Relative expression ratios of $>2.0$ (telomerase, c-KIT, ERBB2) or $>1.0$ (EGFR) in enriched CTC preparations were considered overexpressed and CTC-positive.

\section{Human data}

We randomly and retrospectively studied medical records of 7 cases of advanced (stage four) ovarian cancer, refractory to all chemotherapy regimens (first or second lines and all referred by their oncologists to be treated with this approach). Two of these cases had germline BRCA mutations. We had obtained appropriate consent forms from the patients for the treatment and for the publication. All compounds (Vitamin C, Quercetin, EGCG) were ordered through FDA approved compounding pharmacies (c GMP certified) and through customized prescriptions. Patients summary and responses are outlined below.

Case 1 (Bernice Y): 48 years old female with history of high grade stage IIIC serous ovarian carcinoma diagnosed in February of 2015, status post laparoscopic surgery, further with radical TAHBSO, omentectomy and right diaphragm stripping, and resection of $7 \mathrm{~cm}$ mass in left ovary, involving rectosigmoid and mesentery, total of 6/23 LNs, involved, status post dose dense systemic taxol and carboplatinum for six cycles from $4 / 15$ to $8 / 15$, (was not a candidate for IP chemo), with severe side effects ( neuropathy) and further progressive disease evident on 5/19/16 scan, therefore switched to Carbo and Doxil, post cycle one, received last on $8 / 1 / 16$, was complaining of brain fog, neuropathy and sleep apnea, as well as episodes of asthma, here referred by her gyn oncology ( Dr Chai), to be evaluated and co-managed at our clinic. Upon arrival, she was reporting diarrhea and significant neuropathy in her face and was asking to withhold the chemotherapy. We encouraged her to continue the current regimen at adjusted dose due to side effects.

She had an abdominal and pelvic CT scan on $7 / 18 / 16$, which showed scattered metastatic implants within the abdomen and pelvis, left periaortic lymphadenopathy, enlarging mesenteric LN, left axillary $\mathrm{LN}$, and left adrenal gland implant (which was reported as new finding) along with anterior mediastinum soft tissue.

Upon evaluation, her BRCA testing had been positive for germline BRCA1 mutation. She had been followed by her oncologist, at City of Hope. Her CA 125 had been rising on treatment.

Her labs showed plasma VEGF reported at 118. CA 125, and $\mathrm{LDH}$, circulating tumor cells (CTC) was positive with ERBB2 and telomerase mutations. Her molecular profiling through Caris lab. showed mutations in SMO, ROS1, P53, PTEN and BRCA1. She was immediately started on epigenetic therapies which she received on daily basis for two weeks, consisting of Green Tea, Vitamin C, and Quercetin. Her labs were repeated after two weeks. It showed significant reduction in her VEGF down to 52 from 118 (measured on 8/23/16). Her CA 125 also dropped from 140 to 49.7 .

Her CA 125 was repeated on 9/21/16 and it was at 17.9.(normal range)

Her c DNA responded with improved mutated allele fraction (MAF) of 0.4 percent to non-detectable at PI3k as well as ARID1. Her CTC showed resolution of telomerase activity compared to Pre-therapy, (measured on 8/26/16).

On 9/15/16 the CTC was repeated which showed only marginally elevated ERBB2 with post therapy response on the altered gene.
She was restaged with a whole-body PET scan and there was a significant response to the combination therapy with reduced sizes or complete resolution of the lesions both in chest, and abdomen and pelvis. The retroperitoneal, para aortic LNs and bladder lesion completely resolved as well as adrenal lesion. Left upper quadrant lesions all shrunk in size (largest from $3.0 \mathrm{~cm}$ to $1.6 \mathrm{~cm}$ ) as well as liver surface $(3.4 \mathrm{~cm}$ to $2.4 \mathrm{~cm}$ ) and left kidney (from $3.0 \mathrm{~cm}$ down to 1.7 $\mathrm{cm}$ ). Mesenteric node decreased from $2.4 \mathrm{~cm}$ to $1.3 \mathrm{~cm}$.

Further she moved in June 2017 and was not able to continue the epigenetic therapies as scheduled. She remained on PARP inhibitor (Niraparib at $300 \mathrm{mg}$ daily). She had cancelled many treatment sessions and further not complied with the treatment plan.

On $7 / 10 / 17$, She was admitted to the hospital for an episode of abdominal distention and partial obstruction, where she was restaged with abdominal CT and her scan showed mixed response with progression specifically, in her retroperitoneal (21 mm from $16 \mathrm{~mm}$ ) and periaortic nodes ( 32 from $24 \mathrm{~mm}$ ), as well as abdominal mesenteric artery LN (28 $\mathrm{mm}$ from $16 \mathrm{~mm}$ ).

She unfortunately did not follow as recommended due to her travel distance and did not receive any therapies for over 6 months and passed away in Feb 2018.

Case 2 (Karen C): 42 years old female with history of high grade serous ovarian carcinoma diagnosed on January 2011 status post hysterectomy and oophorectomy. At the time of surgery, she was found to have peritoneal carcinomatosis, and omental involvement, she also has been positive for BRCA2 germline genetic mutation. She received chemotherapy, taxol and carbiplatinum started right after the resection in January and finished on May 2011 ( 2 cycles of Carboplatinum every three weeks and weekly taxol) with biweekly Avastin since 2/2011, further on q 21-day schedule which was scheduled to be continued until May 2012. The tumor marker had been normalized and stayed in normal range, until September 2011, that CA 125 started to rise.

She was followed by Palo Alto and Stanford Women's cancer center and referred to us for evaluation and treatment.

On arrival she was symptomatic with nausea and vomiting. Her labs indicated increased insulin like growth factor one (IGF-1) at 302 and elevated CA 125 of 41.7, measured on 11/4/2011. She was immediately started on targeted epigenetic and antioxidant IV therapies in a protocol on daily basis, four times a week. Her IGF-1 dropped from 302 to 227 after receiving three treatments. Her CA 125 dropped slightly at 40.6 and her vomiting stopped.

Her Natural killer cell activity increased from 36 to 85.3 between $11 / 10 / 2010$ and $2 / 8 / 2012$. Her IGF-1 dropped further to 27 on $2 / 23 / 2012$ (from 302 ) receiving treatments on a biweekly program. Her IGF-1 remained normal measured all through the 2013.

On 4/11/2012 her PET scan was repeated and showed slight improvement in her tumor size and activity compared to $1 / 5 / 2012$. Her CT scan was repeated again on 5/4/2012 that confirmed the complete response to the treatments, and it reported: "no masses are identified. No evidence of any mass or metastatic disease is identified." Also, her CT scan on $8 / 21 / 2012$ confirmed resolution of what appeared to be small pulmonary nodules in the prior PET. The nature of such nodules was described as nonspecific. Her PET scan on 9/18/2012 showed that she had no residual of tumor in her abdomen and pelvis. She continues the treatment per schedule and her CA 125 was 52 on January 14 and 35.4 on January $20^{\text {th }}$ and 76.5 on $3 / 31 / 2012$. Her tumor marker (CA 
Nezami M (2019) Preclinical and preliminary clinical reports for successful treatment of ovarian cancer with standard treatments in combination with epigenetic modifiers in a complimentary and integrative approach

125) continuously dropped to 44.2 measured on $10 / 18 / 2012$ status post weekly therapy of epigenetic treatments on maintenance. Finally, her CA 125 normalized on 11/9/2012 at 34.5. Final read in December 2012 was at 20.

Her scan on December 2012 showed:

1. Significant interval decreases in size and number of bilateral pulmonary nodules now with about 6 remaining nodules compared to about 15 previously. Slight decrease in size of right hilar lymph node.

2. Interval decrease in pelvic fluid with persistent findings of peritoneal nodularity (unchanged $7 \mathrm{~mm}$ ) in the pelvis indicating peritoneal metastases.

3. No new adenopathy or masses.

In July 2012, she decided to start PARP inhibitor trial in Stanford as it was advised merely based on her tumor markers, by her oncologist. This is when her scan continued to show response to therapy only.

She started the trial and received Veliparib per protocol on April 2012, $800 \mathrm{mg}$ a day. Patient received veliparib PO BID on days 1-28. Courses repeat every 28 days in the absence of disease progression or unacceptable toxicity.

She started to respond to the therapy as she continued the program with us. She tried two times to quit our program and each time, her CA 125 increased, suggesting that the combination therapy was effective. She showed resistance to PARPI in December 2012 and had to stop the trial at Stanford.

She returned to receive Epigenetic treatments with us, as she had significant progression of disease on Stanford trial. Upon reinitiating the treatments, her labs improved, Serum IL-8 dropped from 196 to 44 after two weeks of therapy.

We further provided continuous therapy twice a week to her, combined with 60 percent $\left(30 \mathrm{mg} / \mathrm{mm}^{2}\right.$ body surface) of Doxil. She received Epigenetic therapies the day before and after the Doxil, and further had shown partial response documented in her PET scan on $9 / 6 / 13$, with decreased activity of all her lesions from 8.9 down to 3.5 in her pelvis and from 4.6 to 3 in her liver. Her VEGF increased with Doxil to 330 measured on $10 / 7 / 2013$ and dropped after epigenetic therapy to 292. Her HE4 also dropped from 1176 in June 2013, to 845 in July, to 398 in August, and to 344 in September, and 318 on October 2013. Her Fibroblast growth factor also decreased from 13 to 3.3 after the therapy (measured on 10/23/13).

She passed her expected survival and lived beyond 5 years (expected in less than 10 percent of cases).

Case 3 (Natalia M): 60 years old female status post diagnosis of papillary serous adenocarcinoma of right ovary at stage 3 confirmed by pathology on 6/28/2011, status post oophorectomy in June 2011, status post chemotherapy started in July 2011, for 12 weeks. She had received the carboplatinum and Taxol, followed by weekly topotecan, last dose in August 2011.

She referred to us with nausea and vomiting which was evaluated with extensive lab work indicating increased CA 125 of 62.9 measured on $10 / 11 / 2011$. Immediately started on targeted epigenetic and antioxidant IV therapies by protocol. Her nausea and vomiting stopped completely, and her tumor markers dropped to 45.4 measured on 10/13/2011 after receiving only three treatments.
She improved clinically and her CA 125 dropped further to 17.9 measured on $10 / 28 / 2011$ and 12 measured on $11 / 18 / 2011$ and 8 on $1 / 9 / 2012$ and stayed at 8.5 on $3 / 8 / 2012$ and 10.1 on $5 / 10 / 2012$. At this time, she was completely off chemotherapy. Also, her serum creatinine improved from 0.44 to 0.57 in this time, indicating improved muscle mass.

Her molecular profiling by Caris Lab. report indicated response to progesterone s she is started on prometrium as well as Vit D3, as her cancer was VDR positive. Her tumor marker stayed at normal level, 15.1 on $9 / 18 / 2012$ after 12 months of therapy and 15.2 on $1 / 15 / 2013$. Her CA 125, Her4, IGF-1, VEGF, all remained in normal range checked on $3 / 2013$.

Her plasma VEGF dropped from 50 to less than 9 in maintenance program. She stayed in remission for over 12 months on such protocol, without the use of chemotherapy.

Case 4 (Wendelin M): 61-year-old female with history of ovarian serous adenocarcinoma diagnosed in $8 / 2008$, and status post oophorectomy and chemotherapy on 10/2008 treated for 6 months with cisplatinum and taxol, then topotecan for maintenance, CA 125, 1700 on diagnosis, 300 post surgery, after chemo was 9 .

Subsequently patient had a recurrence presenting with bowel obstruction in fall of 2009, and bowel resection in 05/2010, restarted on chemotherapy and developed severe allergic reactions to Carboplatinum and Avastin, changed to cis platinum which she received until December 2010. She was referred by her oncologist, Dr McKee for complimentary treatments to us. (Her oncologist had requested us to treat her and personally accompanied her during her treatment sessions at our office, as she was her relative).

On initial examination she revealed to be cachectic and had lost weight. She was complaining of nausea and loss of appetite along with abdominal pain that was experienced dull and constant. Her abdomen/ pelvic CT scan was performed just before starting the therapies at our facility which revealed suspicious peritoneal metastasis in size of 14X34 $\mathrm{mm}$ along with pleural effusion.

She started the IV epigenetic and antioxidant therapy combined with hyperbaric oxygen treatments on 11/28/2011. Over all she received 11 treatments in two weeks, until 12/9/2011. She reported good sleep and function after the first treatment, no side effects reported. After completing two weeks of therapy her appetite had increased significantly and she gained $5 \mathrm{lbs}$. Her abdominal pain was resolved.

She underwent a full evaluation including blood testing for tumor markers, C reactive protein and natural killer cell activity. Her HE 4 tumor markers dropped from 285 to 255 (and further to 190) after only five treatments. Her CRP also dropped from 4 to 2.4 (normalized) and her WBC increased from 3.3 to 4.1 (normalized) and her hemoglobin increased from 9.8 to 10 only with receiving three treatments between $12 / 1$ to $12 / 5$.

She has expressed significant improvement in her quality of life and pain control/ appetite. Her oncologist further decided to start her back on chemotherapy again in an attempt for complete remission. She moved to northern California and continued her care at her oncologist office implementing the epigenetic therapies.

Patient positive impact in quality of life along with neoplastic response is a desirable approach to modify the current methods.

Case 5 (Audrey F): 55 years old female with history of serous papillary adenocarcinoma of ovaries (and malignant ascites) that was 
Nezami M (2019) Preclinical and preliminary clinical reports for successful treatment of ovarian cancer with standard treatments in combination with epigenetic modifiers in a complimentary and integrative approach

resected on September 2011, in an extensive surgery along with her omentum, (describing surgical report as miliary disease involving all peritoneum) status post 12 cycles of chemotherapy with every three weeks taxol and carboplatinum, started in October 2011, last treatment in June 2012, status post recurrence in less than a month after completing the chemo for 9 months. CA 125 had been increasing very dramatically in the last few months from 85 to 1100 in August 2012.

Unfortunately, the outcome of these tumors after surgical recurrence is not good as she has been given no curative option and even her oncologist had offered no further therapy. She has been given different opinion about the second line neoadjuvant therapy this time again because she had failed the first line chemotherapy. Her PET scan in August showed extensive abdominal cancer in stomach, liver and mesentery, correlating with her surgical findings of peritoneal carcinomatosis. On initial evaluation her labs were drawn which showed extensively high tumor makers CA 125 at 2723 and HE-4 at 1428, increased VEGF at 138 and CRP at 54.

She immediately started the IV epigenetic therapy along with HBOT at our clinic. Her CA 125 was increased 240 percent from august 21th to September 19 (in 4 weeks) before starting the treatments here. After receiving 8 treatments in two weeks, labs were repeated which showed decreased CA 125 from 2723 to 2470 . This decrease happened in less than a two-week period on $10 / 1 / 2012$. This is where the markers were increased 60 percent per week before starting the treatments. In conclusion, the velocity of tumor marker dropped 70 percent from expected number, just in matter of two weeks, (where CA 125 half-life is about two weeks.) Patient was scheduled to proceed with HIPAC therapy that was scheduled in Cancer Treatments Center of America in Oakland and did not return.

Case 6 (Gloria L): 74 years old female with history of carcinosarcoma diagnosed in 2006 status post-surgical resection, and chemotherapy with Taxol, Adriamycin and cisplatinum and carbiplatinum in 2007 status post relapse in 2011 in abdominal cavity, status post resection of the omentum, small bowel, gall bladder, peritoneum, and colon, on $12 / 13 / 2011$.

She was not able to continue chemotherapy due to toxicity (severe neuropathy she experienced in her hands) and she was referred to us for evaluation on 4/11/2012. After initial evaluation which included assessment of her $\mathrm{CBC}$ and $\mathrm{Nk}$ activity, she was started on daily IV epigenetic therapies. She immediately reported significant improvements in her neuropathy and her function. Her CBC before starting the treatments revealed a WBC of 2.6 and her NK activity came back at 4 (normal range 50-300)

After receiving 6 treatments, she was reevaluated. Her CBC was repeated which showed improvement.

She underwent a whole-body PET scan on 4/16/2012 that confirmed resolution of her malignant retroperitoneal lymph nodes and no evidence of recurrent or residual disease.

Case 7 (Stephanie S): 34 years old female with history of high grade serous ovarian adenocarcinoma diagnosed in December 2013 status post few months of abdominal pain treated as IBS! her initial diagnosis was made after an abdominal ultrasound and CT, confirming the presence of two multilobular mixed cystic large right adnexal masses each about 6-9.7 cm in size, along with left upper quadrant and right colon too numerous to count lesions consistent with diffuse peritoneal mets, with possible L4 mets, status post exploratory laparatomy and sampling/ debulking in 1/21/14.
She was unable to urinate which necessitated foley catheterization and emptying of $1400 \mathrm{cc}$ of urine. The nature of the symptom is still unclear but can be realted to caudau equina, as she has a L4 mass in her last PET in 2/14. Initial labs revealed Cyfra 21.1 at 2.9, CA 125 at 1723, HE 4 at 1264, Interleukin 8 at 110, Nk less than 0.2 .

She received carboplatinum and taxol on 5/23/14 and her labs were repeated which showed improved CA 125 but substantially increased interleukin 8 both in serum as well as plasma. Interleukin 8 increased to 286 on $5 / 28 / 14$.

She started the treatments immediately at our clinic with daily IV epigenetic therapies which she received for a total of 10 treatments between 5/12 and 6/18/14. Her labs were repeated and showed significant improvement in her markers as well as interleukin 8 . Her CA 125 dropped to 642 , her HE 4 dropped to 441 and her interleukin 8 (serum) dropped to 46, and plasma normalized at 30 from 286.

\section{Conclusion}

Preclinical and clinical data presented here support the responsible use of natural epigenetic modifiers (in BRCA positive and negative) refractory stage four ovarian cancer. Such approach in treatment of ovarian cancer is considered safe and effective that can be validated in larger studies to be applied as part of the standard treatment offered in recurrent, relapsed cases. We propose continued research and further hypothesis to support expanded knowledge in this area of medicine.

\section{Acknowledgement}

\section{Bharali, PhD.}

\section{References}

1. Winyoo Chowanadisai, Shanta M. Messerli, Daniel H. Miller, Jamie E. Medina, Joshua W. Hamilton, et al. (2016) Cisplatin resistant spheroids model clinically relevant survival mechanisms in ovarian tumors. PLoS One 11: e0151089. [Crossref]

2. James Haley, Sunil Tomar, Nicholas Pulliam, Sen Xiong, Susan M. Perkins, et al. (2016) Functional characterization of a panel of high-grade serous ovarian cancer cell lines as representative experimental models of the disease. Oncotarget 7: 32810-32820. [Crossref]

3. Wilson, L Qiu, Y Hong, T Karnik, G Tadros, et al. (2017) The histone demethylase KDM4B regulates peritoneal seeding of ovarian cancer. Oncogene 36: 2565-2576. [Crossref]

4. Zhang X, Li H, Yu X, Li S, Lei Z, et al. (2018) Analysis of circulating tumor cells in ovarian cancer and their clinical value as a biomarker. Cell Physiol Biochem 48: 19831994. [Crossref]

5. Yunlan Zhou, Bingxian Bian, Xiangliang Yuan, Guohua Xie, Yanhui Ma, et al. (2015) Prognostic value of circulating tumor cells in ovarian cancer: a meta-analysis. PLoS One 10: e0130873.

6. Gerhard Gebauer, Malgorzata Joanna Banys-Paluchowski, Hans Neubauer, Natalia Krawczyk, Anna Kaczerowski, et al. (2017) Clinical relevance of circulating tumor cells in ovarian, fallopian tube and peritoneal cancer. Journal of Clinical Oncology 35.

7. Haller J, Smith, J Michael Straughn, Donald J. Buchsbaum, Rebecca C. Arenda (2017) Epigenetic therapy for the treatment of epithelial ovarian cancer: A clinical review. Gynecol Oncol Rep 20: 81-86. [Crossref]

8. Sara Moufarrij, Monica Dandapani, Elisa Arthofer, Stephanie Gomez, Aneil Srivastava, et al. (2019) Epigenetic therapy for ovarian cancer: promise and progress. Clinical Epigenetics 11:7.

9. Julius strauss, William d. figg (2016) Genitourinary malignancies branch, center for cancer research, national cancer institute, national institutes of health, anticancer research. Chemotherapy Resistance 36: 4-11.

Copyright: (C2019 Nezami M. This is an open-access article distributed under the terms of the Creative Commons Attribution License, which permits unrestricted use, distribution, and reproduction in any medium, provided the original author and source are credited. 